\title{
A Phase-Field Model for High Anisotropic Interfacial Energy
}

\author{
G. B. McFadden" \\ J. J. Eggleston ${ }^{\mathrm{b}}$ \\ P. W. Voorhees \\ U. S. DEPARTMENT OF COMMERCE \\ Technology Admınistratıon \\ Mathematical and Computational Sciences Division \\ Natıonal Institute of Standards \\ and Technology \\ Galthersburg, MD 20899-8230

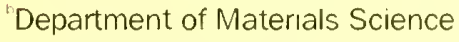 \\ and Engineering \\ Northwestern University \\ Evanston, Illınoıs 60208-3108
}

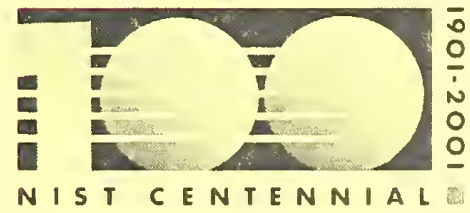

\section{NLI}

National Instifute of Standards and Technology

Technology Administration

U.S. Department of Commerce 


\section{A Phase-Field Model for High Anisotropic Interfacial Energy}

\section{G. B. McFadden ${ }^{\mathrm{a}}$ \\ J. J. Eggleston ${ }^{\mathrm{b}}$ \\ P. W. Voorhees ${ }^{b}$}

U. S. DEPARTMENT OF COMMERCE

Technology Administration

Mathematical and Computational Sciences Division

Natıonal Institute of Standards

and Technology

Galthersburg, MD 20899-8230

Department of Materials Science

and Engineerıng

Northwestern University

Evanston, Illınols 60208-3108

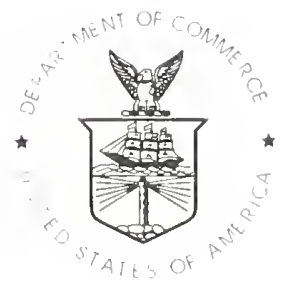

U.S. DEPARTMENT OF COMMERCE

Donald L. Evans, Secretary

NATIONAL INSTITUTE OF STANDARDS

AND TECHNOLOGY

Dr. Karen H. Brown. Actıng Director 


\title{
Phase-Field Model of Anisotropic Surface Free Energy
}

\author{
J.J. Eggleston, G.B. McFadden*, P.W. Voorhees \\ Department of Materials Science and Engineering, Northwestern University, Evanston, Illinois \\ $60208-3108$, UTSA
}

(July 20, 2000)

\begin{abstract}
Anisotropic surface free energy is included in a phase-field model to determine the equilibrium shapes of a free particle in a matrix. Our model allows for a crystal-melt interface with sharp corners due to a highly anisotropic surface free energy with missing orientations. Numerical simulations for various degrees of anisotropy were calculated and they show excellent agreement with analytical equilibrium shapes.
\end{abstract}

\section{INTRODUCTION}

Phase-field models provide a numerical technique that has been shown to be useful for the study of solidification processes [1-7]. The fundamental component of a phase-field model is the inclusion of an additional variable $\phi$, which allows one set of thermodynamic equations to describe a system of multiple phases. The phase field is a constant value in each bulk phase, e.g. $\phi=0$ in the matrix and $\phi=1$ in the particle. The interface between phases is represented by a smooth transition region where $\phi$ varies from zero to one. Modifications are made to the governing thermodynamic functions with the addition of gradient energy

*NIST, Gaithersburg, Maryland 20899-3460 
terms that contribute to the surface energy of the system. The most significant advantage of a phase-field model is the avoidance of explicit tracking of the interface between phases.

The incorporation of surface free energy anisotropy in these models has been considered previously [2, 4, 8-10]. The work of both Hobayashi [2] and Wheeler, Murray, and Schaefer [9] show that anisotropy could be introduced by allowing the gradient energy coefficient to depend on the normal to the interface. McFadden $e t$ al. [4] followed this with an asymptotic analysis for a surface free energy that varies smoothly with orientation, i.e. without missing orientations, and they recovered the Gibbs-Thomsonequation in the sharp-interface limit. A direct link between sharp and diffuse surface motion laws with anisotropy was determined by Taylor and Cahn [11]. They showed the dependence of the energy on surface normal direction was identical with a phase-field or sharp interface model. Fierro et al. [10] performed numerical simulations of an anisotropic phase-field model and the effect which the anisotropy has on the numerical stability. They investigated nonconvex reciprocal anisotropy and the effect convexification had on the motion of a nonconserved phase-field. They did not investigate a conserved evolution equation or the resulting equilibrium solutions. The dynamics of the nonconvex problem should also be investigated in correlation with the numerical technique.

Our aim is to introduce anisotropy into a simple phase-field model for a pure crystal in a liquid melt which can predict equilibrium shapes with sharp corners. When the degree of anisotropy is high enough, orientations disappear from the surface, i.e. corners are developed and the numerical solution of the phase-field equations becomes problematic. We developed a method that is numerically stable for highly anisotropic systems and shows excellent agreement with known equilibrium sliapes.

\section{EQUILIBRIUM SHAPES OF PARTICLES IN A MATRIX}

The effect of anisotropic surface free energy on the equilibrium shape of two-dimensional domains of a phase in a matrix can be described by the Gibbs-Thomsonequation, 


$$
\mu=\mu_{0}+v_{m}\left(\gamma+\gamma_{\theta \theta}\right) K^{\prime}
$$

where $v_{m}$ is the molar volume, $K^{\prime}$ is the curvature, $\gamma$ is the surface free energy and the subscripts denote differentiation with respect to 0 . the angle made by a unit vector normal to the interface and the $x$ axis. Equilibrium is achieved when the chemical potential, $\mu$ is constant throughout the system. Setting $\mu=\mu_{\epsilon}$, a constant, equilibrium shapes can be found by solving Eq. 1 in paranetric form [12]:

$$
\begin{aligned}
& x=\frac{v_{m}}{\mu_{e}-\mu_{0}}\left(\gamma \cos \theta-\gamma_{\theta} \sin \theta\right) \\
& y=\frac{v_{m}}{\mu_{e}-\mu_{0}}\left(\gamma \sin \theta+\gamma_{\theta} \cos \theta\right)
\end{aligned}
$$

for the quadrant $0 \leq \theta \leq \pi / 2$.

The $\theta$ dependence of the surface free energy is unspecified to this point. We have chosen the following $\gamma$ function,

$$
\gamma(\theta)=\gamma_{0}\left(1+\epsilon_{4} \cos 4 \theta\right)
$$

for a crystal with four-fold symmetry. The degree of anisotropy is set by the constant $\epsilon_{4}$ on the interval $[0,1)$. As the anisotropy is increased, the crystal shape will be energy minimizing when certain high energy orientations are missing from the equilibrium shape. Missing orientations occur when the reciprocal $\gamma$ plot first becomes concave [13]. In two dimensions, concavity of $1 / \gamma$ requires that,

$$
\gamma+\gamma_{\theta \theta}=1-15 \epsilon_{4} \cos 4 \theta=0
$$

for some orientations. Thus, missing orientations occur for $\epsilon_{4}>1 / 15$. The first missing orientation $\theta_{m}$, for a given anisotropy can be determined by setting $y=0$ in Eq. 2. The shapes obtained from Eq. 2 include metastable and stable orientations on the "ears" that do not belong to the equilibrium crystal. Mullins [14] proved that in 2-D the equilibrium shape is given by the convex shape remaining after removal of these "ears". The resulting equilibrium shapes for various values of $\epsilon_{4}$ are shown in Fig. 1. 


\section{FIGURES}

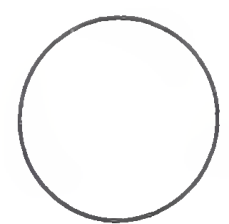

(a) $\epsilon_{4}=0.00$

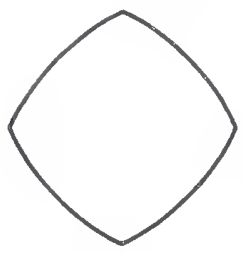

(c) $\epsilon_{4}=0.10$

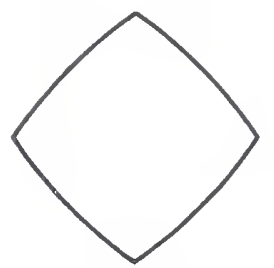

(e) $\epsilon_{4}=0.20$

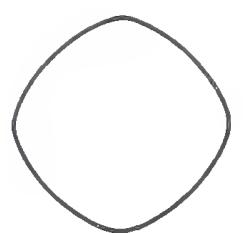

(b) $\epsilon_{4}=0.05$

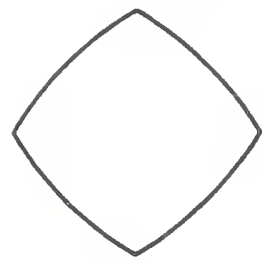

(d) $\epsilon_{4}=0.15$

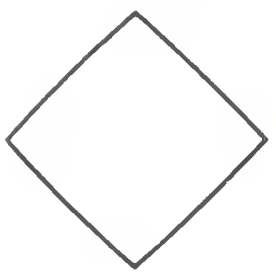

(f) $\epsilon_{4}=0.50$

FIG. 1. Equilibrium shapes for anisotropic crystals with various values of $\epsilon_{\mathbf{4}}$.

\section{DEVELOPMENT OF THE MODEL}

Phase-field models are natural extensions of the diffuse-interface models of Cahn and Allen [15, 16], Ginzburg and Landau [?], and of Cahn and Hilliard [17, 18]. For a single component crystal, the phase-field equations are developed from the free-energy functional [1],

$$
\mathcal{F}=\int_{\Omega}\left(f(\phi)+\frac{\epsilon^{2}}{2}|\nabla \phi|^{2}\right) d \Omega
$$

on the region $\boldsymbol{\Omega}$. The energy density function,

$$
f(\phi)=\frac{W}{4} \phi^{2}(1-\phi)^{2}
$$

is a double-well which has minima at $\phi=0$ and $\phi=1$. With this choice of the free-energy density, the barrier height of the double well potential is $W / 64$. Requiring the free-energy 
functional to decreases monotonically in time for a conserved variable results in the evolution equation,

$$
\frac{\partial \phi}{\partial t}=\nabla \cdot\left(M(\phi) \nabla \frac{\delta \mathcal{F}}{\delta \phi}\right)
$$

where $M(\phi)$ is the mobility and the $\phi$ dependence allows for a mobility that is different in each phase or at the interface.

There are threee steady state one-dimensional solutions of Eq. 7. The first two are of a single-phase $\alpha$ ( $\phi=0$ matrix $)$ or $\beta$ ( $\phi=1$ particle) everywhere in the domain. The third solution is for a planar interface,

$$
\phi(x)=\frac{1}{2}\left[1-\tanh \left(\frac{x}{2 \delta}\right)\right]
$$

where the interfacial thickness,

$$
\delta=\epsilon \sqrt{\frac{2}{W}}
$$

is a balance between two opposing effects. The interface tends to be sharp in order to minimize the regions where the free-energy density is positive which occurs when $\phi$ is between 0 and 1 . Conversely, the interface tends to be diffuse to reduce the energy associated with the gradient of $\phi$. The phase field varies from 0.1 to 0.9 over a distance of $4 \delta$. The excess free energy of the interface is related to the gradient energy coefficient,

$$
\gamma=\frac{\epsilon}{6} \sqrt{\frac{W}{2}}
$$

for the free-energy functional in Eq. 5 with Eq. 6 [16].

To describe anisotropic surface energies, we allow the gradient energy coefficient, $\epsilon$ to depend on the angle of the normal to the contours of constant $\phi[4,5]$. This angle,

$$
\theta=\tan ^{-1}\left(\frac{\phi_{y}}{\phi_{x}}\right)
$$

is the same as the orientation angle described in Section II. Any anisotropy present in $\epsilon$ will appear in the surface energy, $\gamma$ in Eq. 10, as well as the interface thickness, $\delta$ in Eq. 9. We choose the anisotropy in $\epsilon(\theta)$ to coincide with the surface free energy in Eq. 3. Thus, 


$$
\epsilon(\theta)=\epsilon_{0}\left(1+\epsilon_{4} \cos 4 \theta\right)
$$

For values of $\epsilon_{4}>1 / 15$, the polar plot of $1 / \epsilon$ is non-convex when $\epsilon+\epsilon_{\theta \theta}<0$. This leads to an ill-posed evolution equation that is backward parabolic for $\theta$ in the range of missing orientations. The range of missing orientations is given by a common tangent construction to the $1 / \epsilon$ plot, see Fig. 2. Considering the vertical tangent on the right side of the figure, the angles at the tangent points are extrema in the abscissa coordinate,

$$
\frac{d}{d \theta}\left(\frac{\cos \theta}{\epsilon(\theta)}\right)=0
$$

which reproduces the torque-balance condition. The corner angle of the equilibrium shape or first missing orientation, $\theta_{m}$ follows from Eq. 13 and therefore stisfies,

$$
\epsilon\left(\theta_{m}\right) \sin \theta_{m}+\epsilon_{\theta}\left(\theta_{m}\right) \cos \theta_{m}=0
$$

Performing this common tangent construction dictates equilibrium or the balance of chemical forces at the corner. Our method also removes metastable orientations which have $\epsilon+\epsilon_{\theta \theta}>0$ but are not on the equilibrium shape. This is a requirement for local equilibrium.

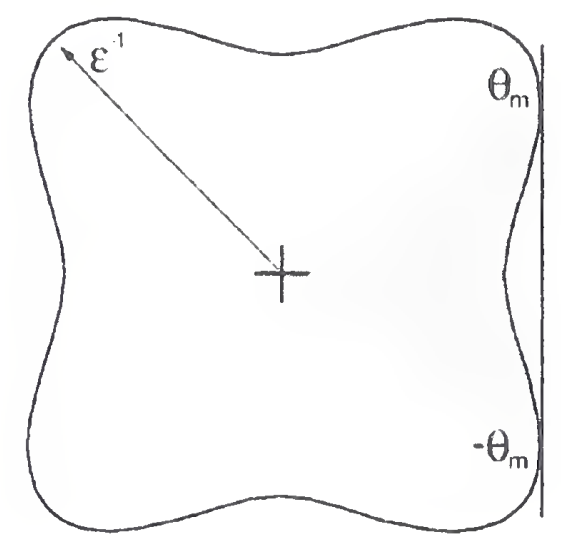

FIG. 2. Convexifying the polar plot of $1 / \epsilon\left(\epsilon_{4}=0.20\right)$.

We propose a regularized gradient energy coefficient, $\tilde{\epsilon}$ using the common tangent to convexify the $1 / \epsilon$ plot. The inversion of the tangent in Fig. 2 with abscissa $\cos \left(\theta_{m}\right) / \epsilon\left(\theta_{m}\right)$ goes into a circle through the origin in the $\epsilon$-plane, see Fig. 3. The convexified portion of 
$\check{\epsilon}$ is thus a portion of the circle with diameter $\epsilon\left(\theta_{m}\right) / \cos \left(\theta_{m}\right)$. Restricting attention to the quadrant $|\theta|<\pi / 4$, the modification to 6 occurs over the range $|\theta|<\theta_{m}$. Our regularized gradient energy coefficient.

$$
\tilde{\epsilon}=\left\{\begin{array}{lc}
\epsilon(\theta) & \text { for } \theta_{m} \leq|\theta| \leq \pi / 4 \\
\frac{\epsilon\left(\theta_{m}\right) \cos \theta}{\cos \left(\theta_{m}\right)} & \text { for }|\theta|<\theta_{m}
\end{array}\right.
$$

will provide a surface free energy anisotropy that has the same equilibrium shapes described in Section II, and in which $\epsilon+\epsilon_{\theta \theta}>0$. Similarly, the expressions for the remaining three quadrants can be determined, reflecting the four-fold symmetry and the piece-wise definition of $\tilde{\epsilon}$.

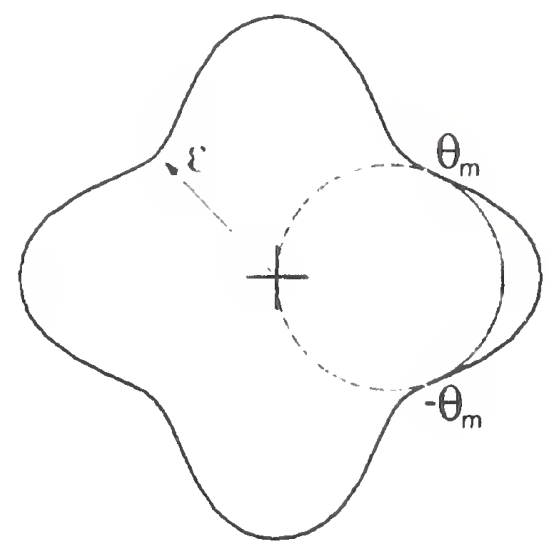

FIG. 3. Polar plot of $\epsilon\left(\epsilon_{4}=0.20\right)$.

Using this convexified gradient energy, Eq. 15 in the free-energy functional, Eq. 5 and using Eq. 7 yields,

$$
\frac{\delta \mathcal{F}}{\delta \phi}=\frac{\partial f}{\partial \phi}-\nabla \cdot\left(\tilde{\epsilon}^{2} \nabla \phi\right)+\frac{\partial}{\partial x}\left(\tilde{\epsilon} \frac{d \tilde{\epsilon}}{d \theta} \frac{\partial \phi}{\partial y}\right)-\frac{\partial}{\partial y}\left(\tilde{\epsilon} \frac{d \tilde{\epsilon}}{d \theta} \frac{\partial \phi}{\partial x}\right)
$$

this is a generalized chemical potential. For non-missing orientations $\left(0_{m} \leq|\theta| \leq \pi / 4\right)$ Eq. 16 can be reformulated into a more suitable form for computations [5],

$$
\begin{aligned}
\frac{\delta \mathcal{F}}{\delta \phi} & =\frac{\partial f}{\partial \phi}-\epsilon^{2} \nabla^{2} \phi-\epsilon \epsilon^{\prime}\left[\sin (2 \theta)\left(\phi_{y y}-\phi_{x x}\right)+2 \cos (2 \theta) \phi_{x y}\right] \\
& +\frac{1}{2}\left(\epsilon^{\prime 2}+\epsilon \epsilon^{\prime \prime}\right)\left[2 \sin (20) \phi_{x y}-\nabla^{2} \phi-\cos (2 \theta)\left(\phi_{y y}-\phi_{x x}\right)\right]
\end{aligned}
$$


where $\epsilon^{\prime}=d \epsilon / d \theta$ and $\epsilon^{\prime \prime}=d^{2} \epsilon / d \theta^{2}$. For missing orientations $\left(|\theta|<\theta_{m}\right)$ Eq. 16 can be simplified greatly due to the specific choice of the convexified gradient energy,

$$
\frac{\delta \mathcal{F}}{\delta \phi}=\frac{\partial f}{\partial \phi}-\left(\frac{\epsilon\left(\theta_{m}\right)}{\cos \left(\theta_{m}\right)}\right)^{2} \phi_{x x}
$$

for the quadrant $|\theta|<\pi / 4$. Sinilar equations can be derived for the remaining three quadrants.

\section{NUMERICAL SIMULATIONS}

Numerical simulations of the anisotropic particle-matrix surface free energy were then performed using an explicit finite difference technique. We solve the evolution equation, Eq. 7 on a two-dimensional uniform grid using a finite difference approximation that is second order accurate in space and first order accurate in time. The mesh spacing is $h$, and the time step $\Delta t$.

\section{A. Equilibrium Shapes}

When the degree of anisotropy is low $\left(\epsilon_{4}<1 / 15\right)$, the equilibrium shape is smooth without corners and the numerical simulation can be performed without difficulty. For the spatial derivatives, simple centered finite differencing formula can be used throughout the domain. The equilibrium shapes that were calculated numerically for $\epsilon_{4}=0.06$ match well with the analytical shapes calculated by Eq. 2 in Section II. Fig. 4 shows this agreement in both the numerical and the analytical equilibrium shapes (shown by the dashed and solid lines respectively). In Fig. 4(a) the dashed numerical equilibrium shape is constructed from a contour of constant phase-field parameter, $\phi=0.5$. In Fig. $4(\mathrm{~b})$ is a polar plot of the surface normal angle as a function of the polar angle of the point on the interface at which the normal angle is measured. 


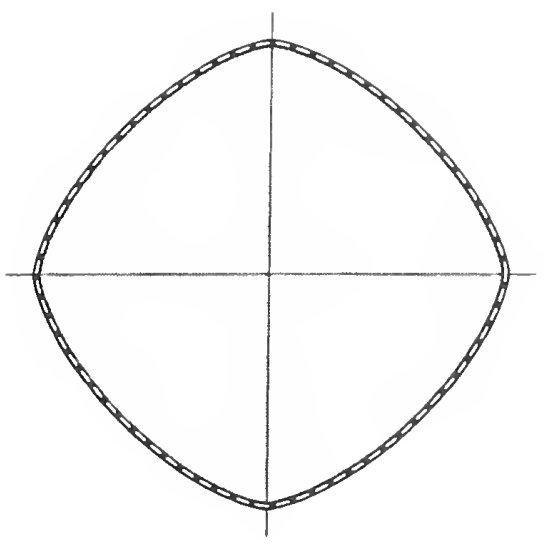

(a) equilibrium shapes

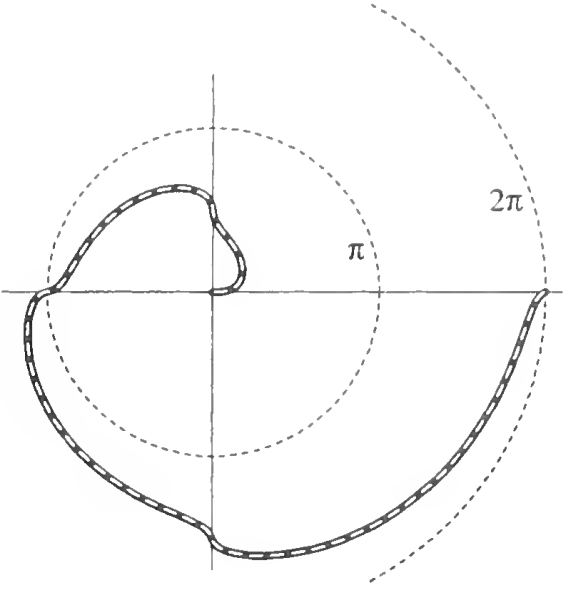

(b) surface normal orientations

FIG. 4. Comparison of numerical dashed line and analytical solid line (a) equilibrium shapes and (b) surface normal orientations, $\theta$ for particles with non-missing surface energy anisotropy $\left(\epsilon_{4}=0.06\right)$.

For anisotropies with $\epsilon_{4}>1 / 15$, orientations disappear from the equilibrium shape and the numerical calculation becomes more involved. First, special care is taken to accurately evaluate $\theta$, the angle of the normal to the contours of constant $\phi$ given by Eq. 11. The first derivatives of $\phi$ across corners are erroneously calculated by centered finite differencing formula. For instance, if a mesh point is located exactly on a corner, then $\theta=0$ using a centered formula (i.e.Eq. 19 and Fig. 5(a)). However, a one-sided formula (i.e.Eq. 20 and Fig. 5(b)) would yield the correct angle on either side of the corner. Although this difficulty can be avoided by rotating the mesh grid relative to the surface anisotropy by $45^{\circ}$ which would reduce the inaccuracy of the centered differencing, this does not completely solve the problem especially for very high anisotropies.

$$
\begin{aligned}
\phi_{y}(i, j) & =\frac{\phi(i, j+1)-\phi(i, j-1)}{2 h} \\
\phi_{y}(i, j) & =\frac{-3 \phi(i, j)+4 \phi(i, j+1)-\phi(i, j+2)}{2 h}
\end{aligned}
$$




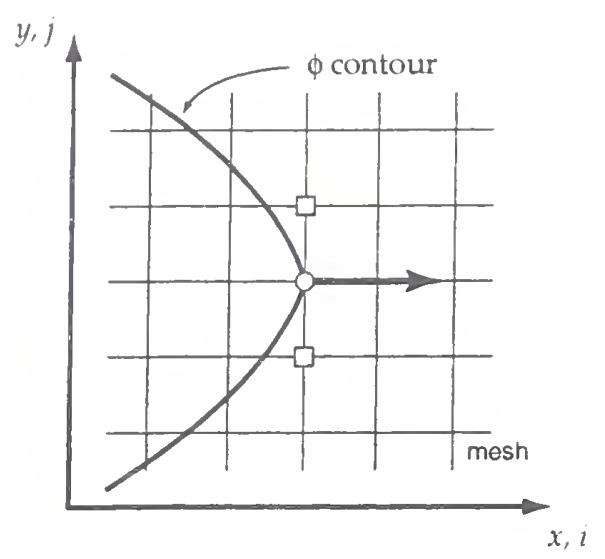

(a) centered

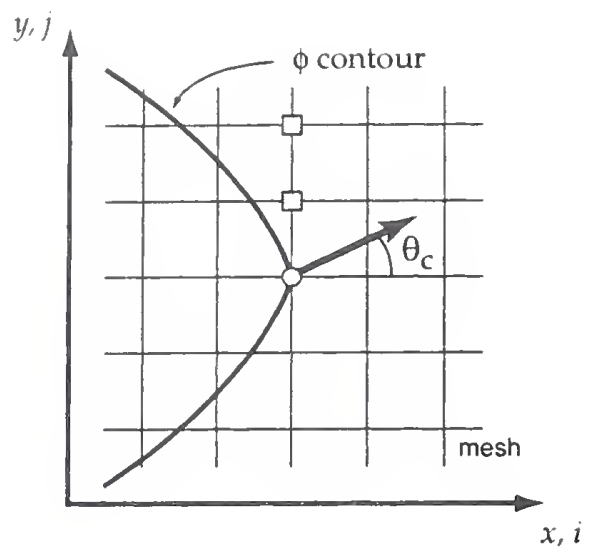

(b) one-sided

FIG. 5. Diagram of $\theta$ calculation by the related first derivatives using (a) centered and (b) one-sided differencing formula across the corner. (where $\theta_{c}$ is the corner angle) In the $x$ direction, centered differencing is used.

The second derivatives $\left(\phi_{x x}, \phi_{y y}, \phi_{x y}\right)$ are also inaccurate using centered differencing formulae near a corner. Unfortunately, using a similar approach as that used in evaluating the first derivatives yields a method that is unstable in time. Ignoring any error caused by using centered second derivatives, we are still able to calculate very good equilibrium shapes. Shown in Fig. 6 is a comparison of the analytical and numerical crystal shapes for $\epsilon_{4}=0.08$ (which is greater than $1 / 15$ and thus has corners). 


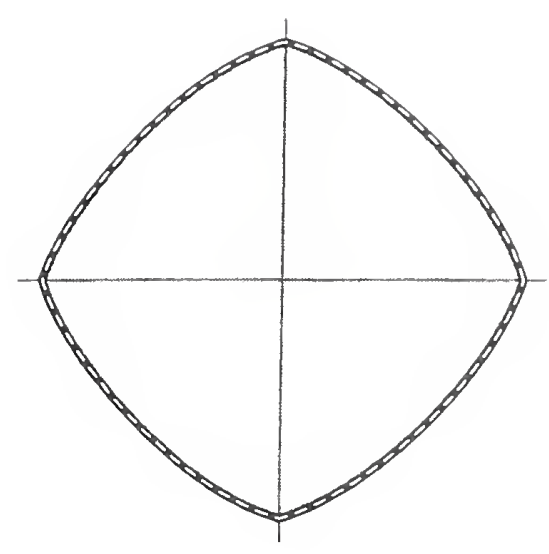

(a) equilibrium shapes

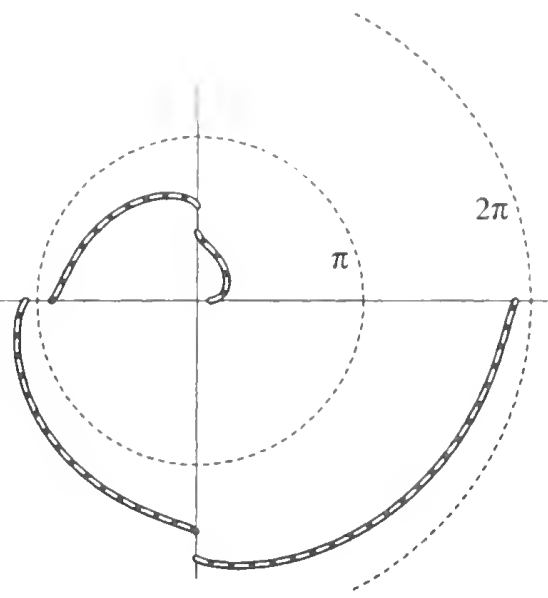

(b) surface normal orientations

FIG. 6. Comparison of numerical dashed line and analytical solid line (a) equilibrium shapes and (b) surface normal orientations $(\theta)$ for particles with missing surface energy anisotropy $\left(\epsilon_{4}=0.08\right)$.

Remarkably, these numerical shapes have discontinuous chemical potentials at the corners, but are still numerically stable. Using centered second derivatives results in a corner chemical potential that changes from sinall to large values nearly every iteration. Completely one-sided second derivatives are accurate, but unstable. Accurately resolving the chemical potential while also maintaining the stability of the numerical method is the trade-off that must be balanced. Thus, a mixed differencing scheme was developed for more accurate calculations near these sharp corners. A comparison between the chemical potentials calculated with this mixed differencing scheme verses that of a simple centered one is shown in Fig. 7. 


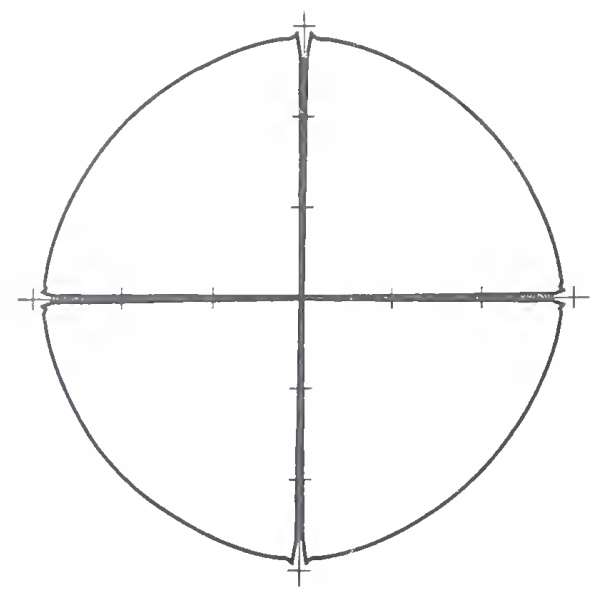

(a) centered

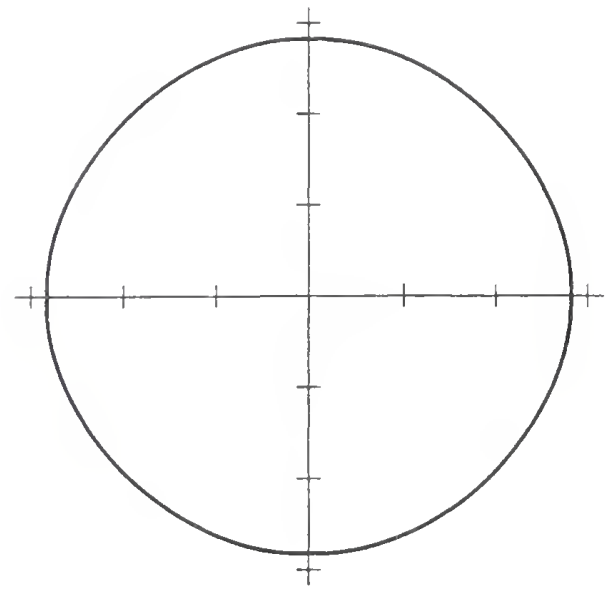

(b) mixed

FIG. 7. Polar polat of the chemical potential along the interface for $\epsilon_{4}=0.08$ using (a) centered and (b) mixed second derivative differencing formula.

In our mixed differencing scheme, the first issue is to locate the corners in our phase-field based on the value of $\theta$ and then choose the differencing scheme for the second derivatives that go across each corner. If $|\theta|<\theta_{m}$, then the convexified gradient energy is used (Eq. 15) and the chemical potential is determined by Eq. 18. The chemical potential only depends on the second $x$ derivative at the corner, but not across it; hence, centered differencing is appropriate. For $|\theta| \gtrsim \theta_{m}$, the full equation for the chemical potential (Eq. 17) must be used. In this region, a weighted average of one-sided and slanted differencing (away from the corner) is used for those derivatives across the corner. The weighted average is 95 to $99.9 \%$ one-sided depending on the anisotropy. If $\theta_{m}<|\theta|<\pi / 8+\theta_{m} / 2$, then a slanted formula is used. Finally for $\pi / 8+\theta_{m} / 2<|\theta| \leq \pi / 4$, centered differencing is used for all derivatives because it is significantly far away from the sharp corner. Examples of differencing formula for centered, slanted, and one-sided are given in Eqs. 21-23.

$$
\begin{aligned}
& \phi_{y y}(i, j)=\frac{\phi(i, j-1)-2 \phi(i, j)+\phi(i, j+1)}{h^{2}} \\
& \phi_{y y}(i, j)=\frac{11 \phi(i, j-1)-20 \phi(i, j)+6 \phi(i, j+1)+4 \phi(i, j+2)-\phi(i, j+3)}{12 h^{2}}
\end{aligned}
$$




$$
\phi_{y y}(i, j)=\frac{2 \phi(i, j)-5 \phi(i, j+1)+4 \phi(i, j+2)-\phi(i, j+3)}{h^{2}}
$$

A similar algorithm, based on the value of $\theta$, for the remaining three quadrants is straightforward.

The chemical potential computed with the centered differencing algorithn is discontinuous but stable, due to the regularization of the gradient energy coefficient. Switching to a mixed differencing algorithm results in a smooth constant chemical potential (Fig. 7). However, the equilibrium shape is unchanged. Computing good equilibrium shapes for values of $\epsilon_{4}<0.5$ is possible with either differencing technique. This limit in surface free energy anisotropy is not due to the finite difference approximation, but instead a result of the mesh resolution. Shown in Figs. 8 and 9 compare the analytical and numerical crystal shapes for $\epsilon_{4}=0.15$ and $\epsilon_{4}=0.30$, respectively.

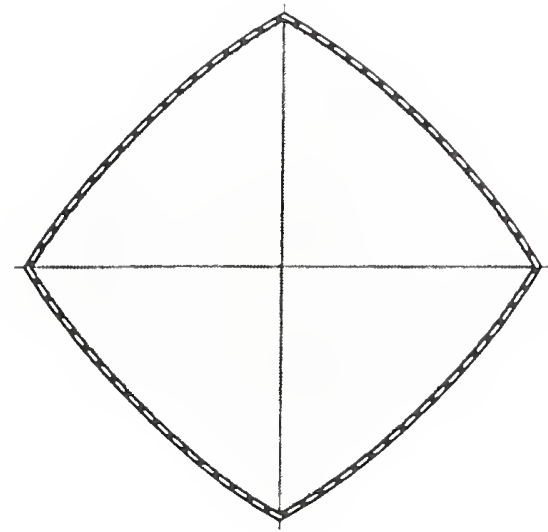

(a) equilibrium shapes

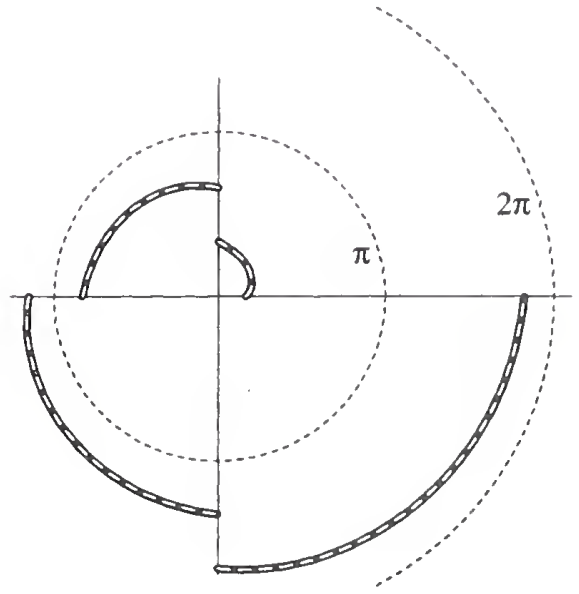

(b) surface normal orientations

FIG. 8. Conıparison of numerical dashed line and analytical solid line (a) equilibrium shapes and (b) surface normal orientations $(\theta)$ for particles with missing surface energy anisotropy $\left(t_{4}=0.15\right)$. 


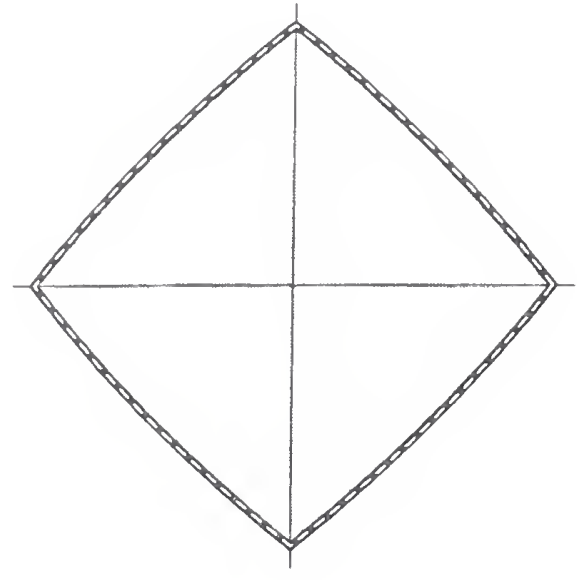

(a) equilibrium shapes

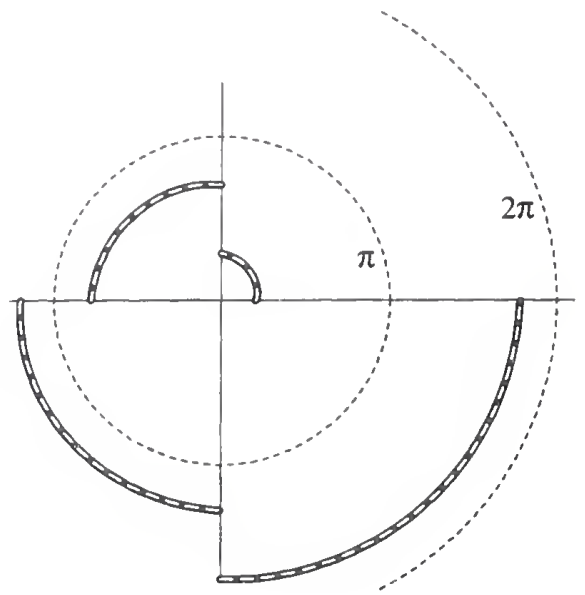

(b) surface normal orientations

FIG. 9. Comparison of numerical dashed line and analytical solid line (a) equilibrium shapes and (b) surface normal orientations $(\theta)$ for particles with missing surface energy anisotropy $\left(\epsilon_{4}=0.30\right)$.

\section{B. Dynamics}

The dynamics of forming crystal shapes with corners from an initially smooth surface was investigated with a centered differencing scheme. The mixed scheme presented above is optimized for shapes that have corners and is inadequate for a circular initial condition. The regions on the initial shape in the missing range of orientations evolve slower than those just outside of this area. The evolution is shown in Fig. 10 by surface contours $(\phi=0.5)$ at various times shown atop each other. This phenomenon is non-physical and is most likely a result of the regular'zed gradient energy. However, Fig. 10 illustrates that the method is stable even though the initial shape had orientations for which $\epsilon+\epsilon_{\theta \theta}<0$. 


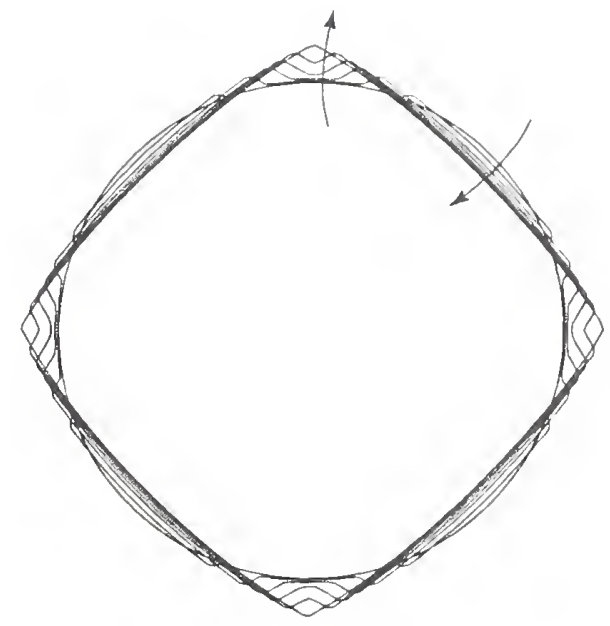

FIG. 10. Surface contours during computation of equilibrium shape $(\epsilon=0.15)$.

Once the corner has formed, its motion is not affected by the differencing scheme. This was tested by tracking the corner positions during the evolution from a rectangular shape to a shape with equal length sides (Fig. 11). No significant difference was found between the computations using centered and mixed differencing formula. Corner motion is unaltered by the discontinuity of the chemical potential present with the centered scheme.

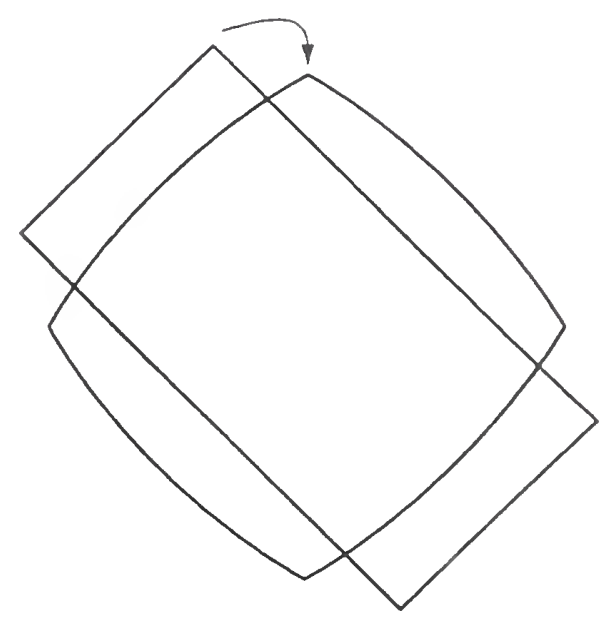

FIG. 11. Diagram of corner motion test, showing the initial and final surface shapes $(\epsilon=0.15)$.

Any difference between the shapes given by a centered or mixed differencing scheme is restricted to one or two mesh points around each corner. These slight differences are not apparent in the equilibrium shapes shown, due to the number of mesh points used for each 
computation $($ mesh $261 \times 261)$. With a centered calculation, the corners are rounded by orientations that should not appear on the equilibrium shape. However, there appears to be no other significant disadvantage with using simple centered differencing for the second derivatives; the time step stability is the same for either method.

A computation was performed on a sinusoidal interface to demonstrate the flexibility of this phase-field method, see Fig 12. The initially smooth surface was imposed with a surface energy anisotropy resulting in corners. Initially the jumps in normal angle or corners develop very quikly. Along the steep slides new corners appear $(t=b)$ due to the enforcement of local equilibrium required by our regularization of the gradient energy. These corners then coarsen $(t=c)$ and disapear $(t=d)$ as the phase-field evolves. The final interface only contains stable orientations. However, it is metastable because the curved surface area can be reduced by coarsening. This computation shows that the method can both introduce new and remove old corners without any implicit tracking of the interface.

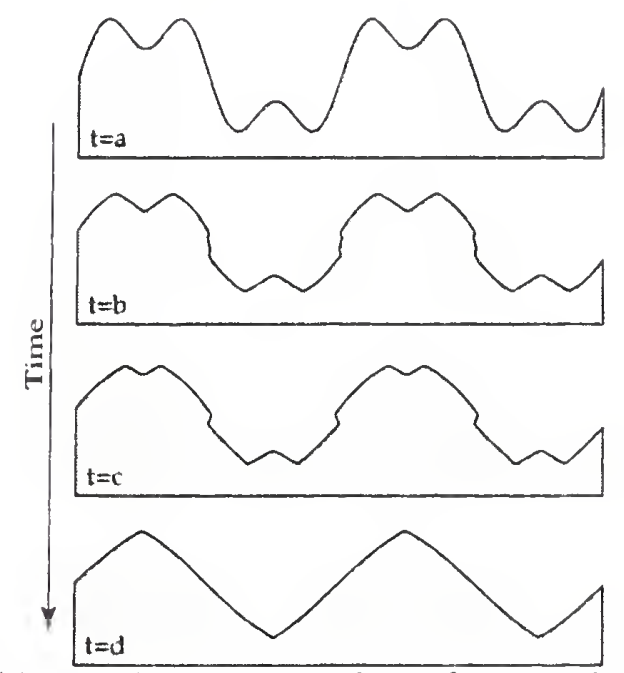

FIG. 12. Surface contours $(\phi=0.5)$ at various times for a periodic sinusoidal computation for $\epsilon 4=0.15$. 


\section{CONCLUSION}

We have introduced anisotropic surface free energy into a phase-field model and computed the equilibrium shapes for crystals both with and without sharp corners. We have shown excellent agreement between our equilibrium shapes and the analytical ones for highly anisotropic particles. With the numerical solution of the phase-field equations for this simple, two dimensional, crystal-melt system, we were able to determine the equilibrium shapes for various degrees of anisotropy. No obvious disadvantage using simple centered differencing for the second derivatives has been determined. Numerical stability, dynamics, and most importantly equilibrium shape are unaffected by the discontinuity of the chemical potential present with the centered algorithm. This validated phase-field method allows similar formulations, for surface energy anisotropy, to be included into more sophisticated phase-field simulations.

\section{ACKNOWLEDGMENTS}

Special thanks goes to NSF and MRSEC for the funding provided for this research. 


\section{REFERENCES}

[1] A.A. Wheeler, W.J. Boettinger, and G.B. McFadden. Phys. Rev. A, 45(10):7424-74.39, 1992.

[2] R. Kobayashi. Physica D, 63:410, 1993.

[3] A.A. Wheeler, W.J. Boettinger, and G.B. McFadden. Phys. Rev. E, 47(3):1893-1909. 1993.

[4] G.B. McFadden, A.A. Wheeler, R.J. Braun, and S.R. Coriell. Phys. Rev. E, 48(3):2016$2024,1993$.

[5] J.A. Warren and W.J. Boettinger. Acta Metall. Mater., 43(2):689-703, 1995.

[6] W.J. Boettinger and J.A. Warren. Mettal. Trans. A, 27A:657-669, 1996.

[7] R. Kobayashi, J.A. Waren, and W.C. Carter. Physica D, 119:415-423, 1998.

[8] G. Caginalp and P. Fife. Phys. Rev. B, 34:4940, 1986.

[9] A.A. Wheeler, B.T. Murray, and R.J. Schaefer. Physica D, 66:243, 1993.

[10] F. Fiero and R. Goglione. Math. Models and Methods in App. Sci., 8(4):573-601, 1998.

[11] J.E. Taylor and J.W. Cahn. J. Stat. Phys., 77:183-197, 1994.

[12] W.K. Burton, N. Cabrera, and F.C. Frank. Trans. Roy. Soc. London, A243:299, 1951.

[13] F.C. Frank. Metal Surfaces. ASM, 1963.

[14] W.W. Mullins. J. Math. Phys., 3:754, 1962.

[15] J.W. Cahn and S.M. Allen. J. Phys. (Paris) Colloq. Suppl., C.7:51, 1977.

[16] S.M. Allen and J.W. Cahn. Acta metall., 27:1085-1095, 1979.

[17] J.W. Cahn and J.E. Hilliard. J. Chem. Phys., 28:258, 1958. 
[18] J.W. Cahn. Acta metall., 9:795, 1961. 

